\title{
An Interdisciplinary Approach to the Management of Individuals With Tracheostomy
}

\author{
Hashem Alhashemi, Mohammed Algarni, Hadi Al-Hakami, Narvanie Seebran, Tanvir Hussain, \\ Tariq Bhutto, Abdulla Tashkandi, Mazen Alayed, Elham Bukhari, and Abdulsalam Alzahrani
}

\begin{abstract}
BACKGROUND: Study objectives were to identify the proportion of tracheostomy subjects with successful decannulation, time to decannulation after ICU discharge, and predictors of long-term tracheostomy based on an interdisciplinary team approach. METHODS: This retrospective cohort study recruited all adult tracheostomy patients admitted between January 2016 and December 2018. Long-term tracheostomy patients with recurrent admissions and compromised airway and patients with neck tumors obstructing the airway were excluded. Data regarding subjects' demographics, comorbidities, Glasgow coma score (GCS), feeding, ICU discharge date, decannulation date, and outcome were collected. The interdisciplinary team members included tracheostomy resource nurse; respiratory therapist; speech clinician; ear, nose, and throat specialist; and rehab medicine specialist. RESULTS: Of the 221 subjects followed during the study period, $16 \%(36 / 221)$ were excluded, and the remaining $84 \%(185 / 221)$ underwent the decannulation protocol. Subjects who failed capping multiple times 114/185 $(62 \%)$ were labeled long term and did not progress to decannulation. We successfully decannulated $71 / 185$ subjects $(38 \%)$, and none developed decannulation failure. Forty deaths occurred during hospitalization, but none was due to tracheostomy complications. The median time to decannulation after ICU discharge was $47 \mathrm{~d}$. Predictors of long-term tracheostomy were GCS $<11$ (odds ratio [OR] 5.6 [95\% CI 2.7-12.0]), age $\geq 65$ y (OR 4.5 [95\% CI (2.1-10.0]), comorbidities $\geq 2$ (OR 4.0 [95\% CI 1.5-11.2]), and female sex (OR 3.0 [95\% CI 1.3-7.4]). The proportion of subjects with long-term tracheostomy significantly increased with the total number of predictors (Fisher exact test, $P<.001)$. CONCLUSIONS: Long-term tracheostomy was a common outcome among subjects with a tracheostomy. Older age, low GCS, female gender, and the number of comorbidities were significant long-term tracheostomy predictors. Further studies to assess outcomes and predictors of tracheostomy are needed. Key words: tracheostomy; airway; intensive care units; teamwork; decannulation; care continuity. [Respir Care 2022;67(1):34-39. (C) 2022 Daedalus Enterprises]
\end{abstract}

\section{Introduction}

Tracheostomy is a common procedure performed in critically ill patients. ${ }^{1}$ This method has several advantages, including decreased airway resistance, improved secretion clearance, and decreased duration of mechanical ventilation and ICU length of stay. ${ }^{2}$ However, tracheostomy is associated with several complications and increased morbidity and mortality. ${ }^{3}$ Tracheostomy patients have increased risks of malnutrition, nosocomial infections, pressure sores, and fistulas. ${ }^{4}$ Furthermore, these patients place a significant burden on hospital resources and account for the highest levels of hospital reimbursement. ${ }^{5,6}$
There are no data from Saudi Arabia regarding the outcomes of patients undergoing tracheostomy. This study aimed to examine the outcomes of tracheostomy subjects under an interdisciplinary team approach. The objectives were to identify the proportion of subjects with successful decannulation, the time to decannulation after ICU discharge, and predictors of long-term tracheostomy.

\section{Methods}

This retrospective cohort study was approved by the institutional review board of the King Abdullah International Medical Research Center. Due to the study's retrospective design, informed consent was not required. 
All adult tracheostomy subjects admitted between January 2016 and December 2018 were included. Long-term tracheostomy patients with recurrent admissions and compromised airway and patients with neck tumors obstructing the airway were excluded.

An interdisciplinary tracheostomy team followed all subjects. To identify variables that could predict decannulation protocol outcomes, subjects who were successfully capped and decannulated were compared to those who failed capping and did not progress to decannulation.

We retrieved data on the subjects' age, sex, comorbidities, Glasgow coma score (GCS), feeding, tracheotomy date, ICU discharge date, decannulation date, and outcome. ${ }^{7,8}$ Comorbidities included diseases commonly encountered in tracheostomy patients (traumatic brain injury, stroke, brain tumors, pneumonia, COPD, heart failure, ischemic heart disease, and end-stage renal disease on dialysis).

The interdisciplinary tracheostomy team consisted of a respiratory therapist; a speech and swallowing clinician; an ear, nose, and throat (ENT) specialist; a rehabilitation medicine specialist; and a tracheostomy resource nurse. The respiratory therapist performed daily tracheostomy tube care; the speech and swallowing clinician performed endoscopic airway assessment and clinical and endoscopic swallowing assessments; the ENT specialist diagnosed and managed airway complications; the rehabilitation medicine specialist evaluated the subjects' cognition and medical comorbidities; and the tracheostomy resource nurse ensured that subjects' needs, regarding caregiver education, training, home supply, and equipment, were met. The team was notified as soon as the tracheostomy subjects were discharged from the ICU or admitted to a general unit. Tracheostomy subjects were discussed during weekly clinical rounds, and

Dr Alhashemi is affiliated with Department of Medicine, Ministry of National Guard-Health Affairs, King Saud bin Abdulaziz University for Health sciences, Jeddah, Saudi Arabia. Dr Algarni and Dr Al-Hakami are affiliated with Department of Surgery, Ministry of National Guard-Health Affairs, King Saud bin Abdulaziz University for Health sciences, Jeddah, Saudi Arabia. Ms Seebran is affiliated with Nursing Education Department, Ministry of National Guard-Health Affairs, Jeddah, Saudi Arabia. Dr Hussain is affiliated with Otolaryngology and Head and Neck Surgery Department, Sligo Unviersity Hospital, Ireland. Mr Tashkandi and $\mathrm{Mr}$ Alzahrani are affiliated with Respiratory Care Services Department, Ministry of National Guard-Health Affairs, Jeddah, Saudi Arabia. Mr Alayed and Dr Bhutto are affiliated with Department of Surgery, Ministry of National Guard-Health Affairs, Jeddah, Saudi Arabia. Dr Bukhari is affiliated with Nursing Education Deparment, Ministry of National Guard-Health Affairs, Jeddah, Saudi Arabia.

The authors declare no conflicts of interest.

Correspondence: Hashem Alhashemi MBBS MPH FRCPC, Department of Medicine, Ministry of National Guard-Health Affairs, King Saud bin Abdulaziz University for Health sciences, PO Box 9515, Jeddah 21423, Saudi Arabia. E-mail: Hashemhh@yahoo.com.

DOI: $10.4187 /$ respcare. 08869

\section{QUICK LOOK}

\section{Current knowledge}

A team approach for delivering care to tracheostomy patients improves their outcomes. Several decannulation predictors have been identified in the literature.

\section{What this paper contributes to our knowledge}

Older age, low Glasgow coma score (GCS), female gender, and the number of comorbidities were significant long-term tracheostomy predictors. The ability to identify patients with multiple predictors for long tracheostomy is essential for effective utilization of resources and proper reporting of teams' outcomes. Subsequently, subjects with $\geq 3$ predictors could be discharged and/or reevaluated when their GCS or comorbidities had improved.

management decisions were based on the tracheostomy decannulation protocol (Fig. 1). ${ }^{8-10}$

Tracheostomy tube with inflated cuff is needed to maintain positive-pressure ventilation; however, after extubation cuff, deflation is needed to progress toward decannulation. Subjects who were vitally stable underwent suctioning and monitoring before and during the trial of tracheostomy cuff deflation.

On the initial assessment by the tracheostomy team (after ICU discharge), most of the subjects had already deflated cuffs. Dried secretions may obstruct the tracheostomy tube and cause respiratory distress; therefore, air humidification is an essential component of care for tracheostomy patients. A heat and moisture exchanger was used for air humidification for tracheostomy subjects in this cohort. In addition, innertube care was performed (1-2 times per shift) for double lumen tracheostomy tubes to prevent tracheostomy tube obstruction due to dried/thick secretions. Subjects who were medically stable, emerged out of coma, had strong cough, and minimal secretions underwent endoscopic airway and swallowing evaluation by the speech and language pathologist. Then, subjects with compromised airway had tracheostomy tube (cuff deflated) changed to cuffless nonfenestrated. On the other hand, subjects with good airway assessment had tracheostomy tube (cuff deflated) change to a cuffless fenestrated tube and progressed to a capping trial. The endoscopic airway evaluation is an anatomical assessment, and the capping trial is a functional/physiological assessment for the airway. Subsequently, subjects who had successful capping progressed to decannulation. Capping failure was defined as respiratory distress and/or the inability to maintain oxygen saturation during a 72-h trial of tracheostomy tube capping. ${ }^{11}$ When subjects failed the capping trial, further capping was delayed; and reassessment of the decannulation protocol steps regarding suctioning/secretions management, medical 


\section{INTERDISCIPLINARY MANAGEMENT OF TRACHEOSTOMY}

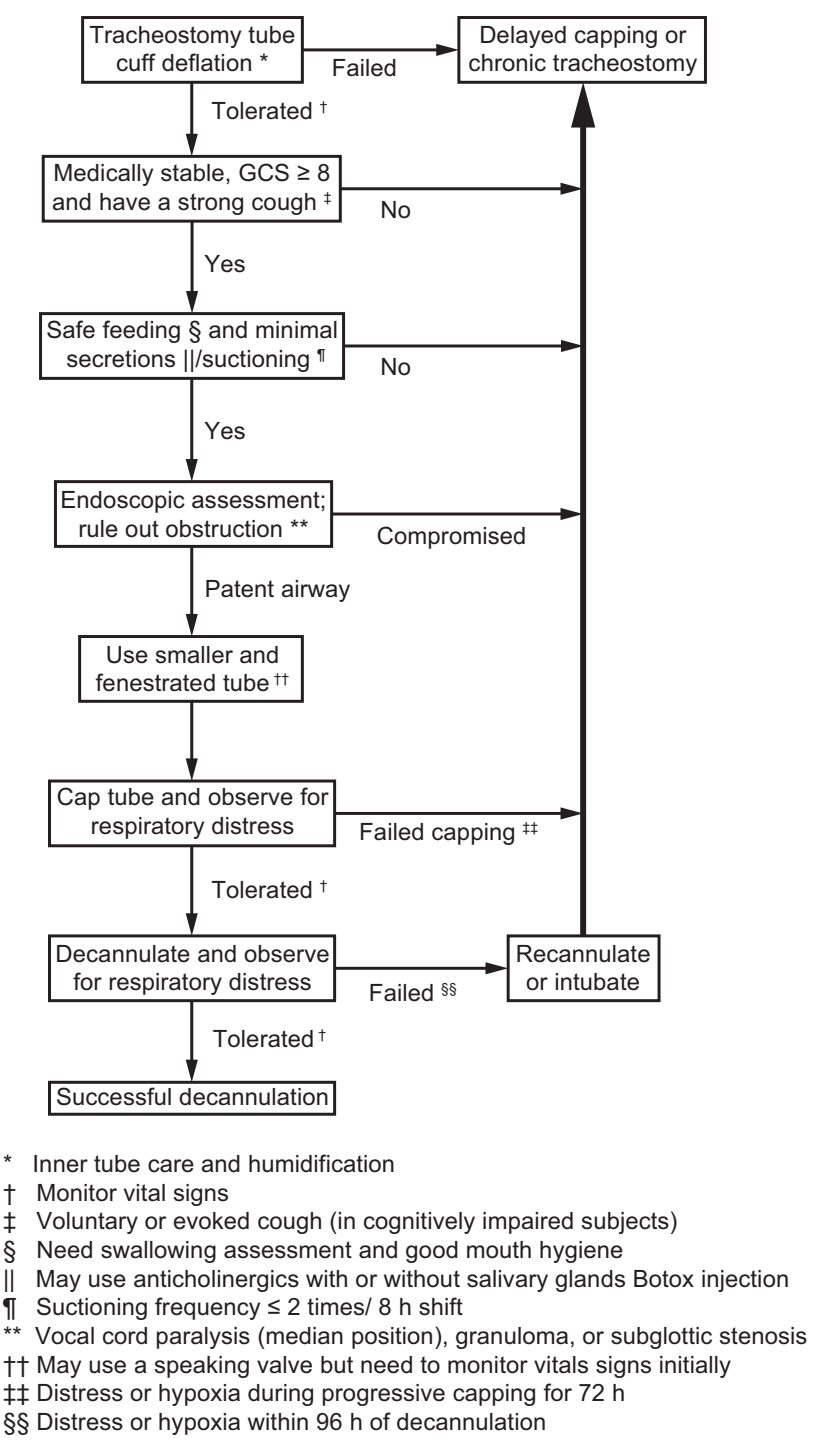

Fig. 1. Tracheostomy decannulation protocol. GCS = Glasgow coma score.

stability, tube type/size, and airway assessment for granulomas/obstructive lesions was repeated. If the reason for capping failure remained unknown, a computed tomography for neck and chest was done to rule out subglottic stenosis, tracheal stenosis, tracheomalacia, and/or tracheoesophageal fistula. Subjects who failed repeated capping trials did not progress to decannulation and were labeled long-term tracheostomy.

The objective of swallowing evaluation was to ensure safe feeding, and the recommended feeding modality did not impact decannulation protocol. Tube-fed subjects who had successful capping underwent a repeated swallowing evaluation after decannulation because normalization of subglottic pressure and laryngeal mobility could improve dysphagia.

Decannulation failure was defined as the need for stoma recannulation or oral intubation due to respiratory distress and/or the inability to maintain oxygen saturation $48-96 \mathrm{~h}$ after decannulation. ${ }^{12,13}$ Based on a small pilot study, the proportion of patients with failed capping was estimated to be approximately 50\%. The subjects' electronic medical records were checked for data availability. Sample size calculation was performed using the formula $n=10 \mathrm{~K} / \mathrm{P}$, where $\mathrm{K}$ is the number of predictors needed in the model and $\mathrm{P}$ is the smallest proportion among positive or negative cases. ${ }^{14}$ The nQuery program was used for sample size sensitivity analysis. After accounting for missing data, the estimated sample size for a power of $80 \%$ and a 2-sided alpha level of $0.05 \%$ was 140 subjects. Data analysis was done using IBM SPSS Statistics Version 26 (IBM, Armonk, New York).

\section{Results}

The team followed 221 subjects during the study period. Of these, $16 \%(36 / 221)$ was excluded as they were longterm tracheostomy subjects with recurrent admissions (range, 2-5 times) and compromised airway, and 84\% $(185 / 221)$ underwent the decannulation protocol (Fig. 1). We successfully decannulated $71 / 185$ subjects $(38 \%)$, and none developed decannulation failure. The median time to decannulation after ICU discharge was $47 \mathrm{~d}$. Subjects who failed capping multiple times, 114/185 (62\%), were labeled long term and did not progress to decannulation.

The subjects' characteristics and outcomes are presented in Table 1. Chi-square and Fisher exact tests showed that sex, comorbidities, age, GCS, feeding, and mortality were significantly associated with decannulation protocol outcomes. The most prevalent comorbidity was brain injuries (stroke, traumatic brain injuries, brain tumors), 78\% (145/185); however, the highest capping failure rates were noted among subjects with ischemic heart disease and/or heart failure, 90\% (14/15), and those with end-stage renal disease undergoing dialysis, $100 \%$ (13/13). Using multiple logistic regression, the significant predictors for long-term tracheostomy among subjects ( $n=185)$ were determined to be GCS $<11$ (odds ratio [OR] 5.6 [95\% CI 2.7-12.0]), age $\geq 65$ y (OR 4.5 [95\% CI, 2.110.0]), comorbidities $\geq 2$ (OR 4.0 [95\% CI 1.5-11.2]), and female sex (OR 3.0 [95\% CI 1.3-7.4]; Table 2). When subjects were grouped according to the total number of predictors $(0,1,2, \geq 3)$, a significant association was found between the number of predictors per subject and the proportion of subjects with long-term tracheostomy (Fisher exact test, $P<.001)$. The proportion of subjects with long-term tracheostomy significantly increased with the total number of predictors (Fig. 2).

Subjects with a long-term tracheostomy were discharged after receiving caregiver education and tracheostomy home care supplies. Fifteen subjects were decannulated during follow-up visits in the tracheostomy clinic after hospital discharge. Of the entire cohort, 18\% (40/221) died. 


\section{INTERDISCIPLINARY MANAGEMENT OF TRACHEOSTOMY}

Table 1. Outcomes and Characteristics of Tracheostomy Subjects*

\begin{tabular}{|c|c|c|c|c|}
\hline Variable & Subjects, $n$ & Decannulated $n(\%)$ & Long-Term Tube $n(\%)$ & $P$ \\
\hline \multicolumn{5}{|l|}{ Age, y } \\
\hline$<65$ & 104 & $57(55)$ & $47(45)$ & \multirow[t]{2}{*}{.001} \\
\hline$\geq 65$ & 81 & $14(17)$ & $67(83)$ & \\
\hline \multicolumn{5}{|l|}{ Sex } \\
\hline Male & 139 & $59(42)$ & $80(58)$ & \multirow[t]{2}{*}{.05} \\
\hline Female & 46 & $12(26)$ & $34(74)$ & \\
\hline \multicolumn{5}{|l|}{ GCS } \\
\hline$\geq 11$ & 81 & $48(59)$ & $33(41)$ & \multirow[t]{2}{*}{.001} \\
\hline$<11$ & 104 & $23(22)$ & $81(78)$ & \\
\hline \multicolumn{5}{|l|}{ Feeding } \\
\hline Oral & 18 & $15(83)$ & $3(17)$ & \multirow[t]{2}{*}{$.001^{\dagger}$} \\
\hline Tube & 167 & $56(34)$ & $111(66)$ & \\
\hline \multicolumn{5}{|l|}{ Comorbidities } \\
\hline$<2$ & 138 & $65(47)$ & $73(53)$ & \multirow[t]{2}{*}{.001} \\
\hline$\geq 2$ & 47 & $6(13)$ & $41(87)$ & \\
\hline \multicolumn{5}{|l|}{ Brain injury } \\
\hline Yes & 143 & $56(39)$ & $87(61)$ & \multirow[t]{2}{*}{.70} \\
\hline No & 42 & $15(36)$ & $27(64)$ & \\
\hline \multicolumn{5}{|l|}{ IHD or heart failure } \\
\hline Yes & 15 & $1(7)$ & $14(93)$ & \multirow[t]{2}{*}{.01} \\
\hline No & 170 & $70(41)$ & $100(59)$ & \\
\hline \multicolumn{5}{|l|}{ COPD or pneumonia } \\
\hline Yes & 41 & $9(22)$ & $32(78)$ & \multirow[t]{2}{*}{.01} \\
\hline No & 144 & $62(43)$ & $82(57)$ & \\
\hline \multicolumn{5}{|l|}{ End-stage renal disease on dialysis } \\
\hline Yes & 13 & $0(0)$ & $13(100)$ & \multirow[t]{2}{*}{.002} \\
\hline No & 172 & $71(41)$ & $101(59)$ & \\
\hline \multicolumn{5}{|l|}{ Hospital mortality } \\
\hline Died & 36 & $2(6)$ & $34(94)$ & \multirow[t]{2}{*}{$.001^{\dagger}$} \\
\hline Survived & 149 & $69(46)$ & $80(54)$ & \\
\hline \multicolumn{5}{|l|}{ Tracheostomy indication } \\
\hline Prolonged ventilation & 158 & $59(37)$ & $99(63)$ & \multirow[t]{2}{*}{.50} \\
\hline Others tracheostomy indication & 27 & $12(44)$ & $15(56)$ & \\
\hline
\end{tabular}

Table 2. Predictors of Long-Term Tracheostomy Using Multiple Logistic Regression

\begin{tabular}{|c|c|c|c|c|c|c|}
\hline \multirow{2}{*}{ Predictors } & \multicolumn{3}{|c|}{ Unadjusted } & \multicolumn{3}{|c|}{ Adjusted } \\
\hline & OR & $P$ & $95 \% \mathrm{CI}$ & OR & $P$ & $95 \% \mathrm{CI}$ \\
\hline Female & 2.0 & .051 & $1.0-4.4$ & 3.0 & .01 & $1.3-7.4$ \\
\hline Comorbidities $\geq 2$ & 6.0 & .001 & $2.4-15.0$ & 4.0 & .007 & $1.5-11.2$ \\
\hline Age $\geq 65 y$ & 5.8 & .001 & $3.0-11.6$ & 4.5 & .001 & $2.1-10.0$ \\
\hline $\mathrm{GCS}<11$ & 5.0 & .001 & $2.7-10.0$ & 5.6 & .001 & $2.7-12.0$ \\
\hline
\end{tabular}

However, no subject died due to tracheostomy complications. There were 4 deaths among the excluded 36 longterm tracheostomy subjects $(11 \%)$ and 36 among the 185 subjects who underwent decannulation protocol (19\%). The difference in mortality between them was not statistically significant (chi-square test, $P=.20$ ).

\section{Discussion}

Tracheostomy patients may have airway, medical, and neurological abnormalities; therefore, multiple disciplines are involved in decannulation. Multidisciplinary and interdisciplinary team approaches are used to manage patients with complex medical conditions. In the multidisciplinary approach, team members work independently, whereas in the interdisciplinary approach team members work together, collaborate, and communicate effectively to manage the patients. ${ }^{15,16}$ A team-based approach when delivering care to 


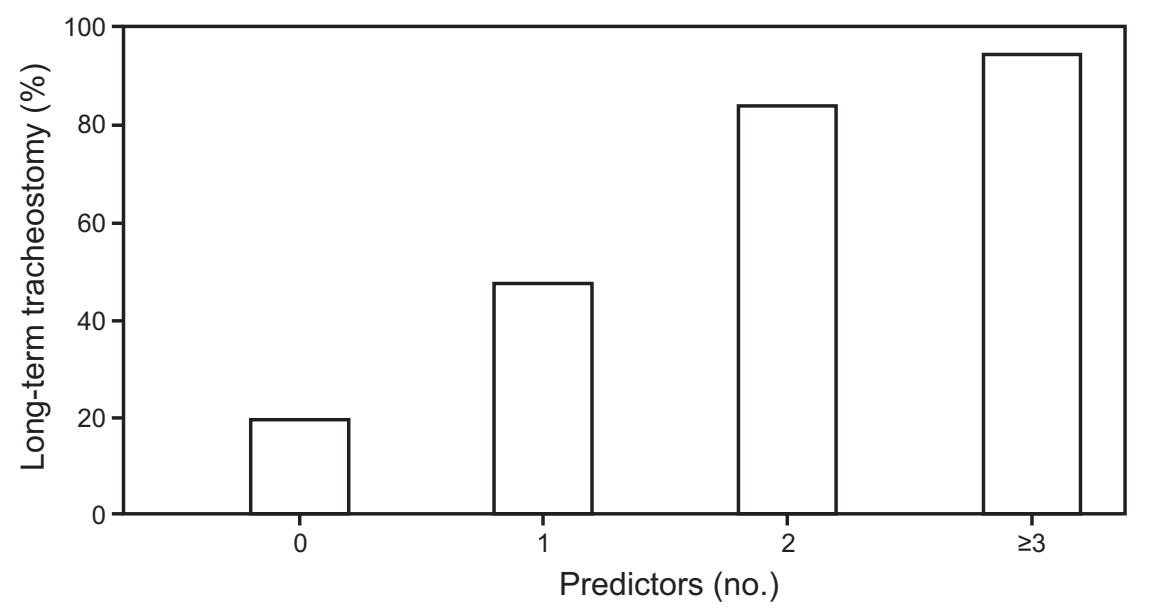

Fig. 2. Association between number of predictors and long-term tracheostomy.

tracheostomy patients improves their outcomes. ${ }^{17}$ The outcomes reported in this study were achieved using the interdisciplinary team approach, which ensured continuity of care, decreased the burden on individual clinicians, bridged experience gaps among team members, and improved the ability to make airway management decisions.

The rate of successful decannulation varies in the literature from $20-80 \%$, depending on the setting and patient population studied. ${ }^{17-21}$ The decannulation failure rate is in the range of $2-5 \% .^{12,13}$ The reported mortality of tracheostomy patients varies between $20-40 \%$, primarily due to significant associated morbidities. ${ }^{3,22,23}$ The time from ICU discharge to decannulation was reported to reflect team performance because total tracheostomy time and length of hospital stay were affected by complications that delayed ICU discharge. Furthermore, hospital length of stay was affected by complications that delayed hospital discharge post-decannulation. ${ }^{24}$ The identified predictors (age, GCS, sex, and comorbidities) were also significant in previous studies ${ }^{9,12,13,17,20,25}$; however, our study was the first to combine them into one model.

Moreover, this study was the first to report that subjects with multiple predictors were more likely to have longterm tracheostomy (Fig. 2). The ability to identify subjects with multiple predictors for long-tracheostomy is essential for effective utilization of resources and proper reporting of teams' outcomes. Subsequently, subjects with 3 or more predictors could be discharged and/or reevaluated when their GCS or comorbidities had improved. ${ }^{26}$

Although decannulation predictors varied in the literature, they are clinically related. For example, the ability to cough is related to the level of consciousness; the need for suction is related to cough strength; pooling of secretions is related to the degree of dysphagia; oxygenation is related to medical stability and comorbidities. ${ }^{27}$ Whereas trying to fit all possible/related predictors into one model is attractive, it is difficult due to reasons like sample size constraints and multicollinearity. On the other hand, identifying the least number of predictors for an outcome is essential and more practical/helpful to clinicians. The identified predictors (age, sex, comorbidities, and GCS) had the advantage of being simple and readily available. Other predictors reported in the literature were not included in this study because of the difficulty in measuring cough effectiveness among comatose subjects (cough peak flow and maximum expiratory pressure), the need for special expertise and equipment (endoscopic airway and swallowing evaluation) that may not be available at every center, and the overlap with studied predictors (respiratory failure and comorbidities). ${ }^{10,11,13,28}$ Note that although all medically stable and cognitively able subjects underwent bedside and instrumental swallowing evaluations by the speech and language clinician only $8 \%$ $(15 / 185)$ could tolerate an oral diet at the time of decannulation (Table 1).

\section{Limitations}

This study's design was retrospective; however, there were no missing data related to the subjects' outcomes or the assessed predictors. This is related to the electronic nature of the records and the completeness of documentation. Moreover, long-term tracheostomy was not a rare outcome (62\%); therefore, the reported OR may have overestimated its risk. Finally, the association between the number of predictors and long-term tracheostomy needs validation in future studies.

\section{Conclusions}

Long-term tracheostomy is a common outcome among tracheostomy patients. Older age, low GCS, female gender, 


\section{INTERDISCIPLINARY MANAGEMENT OF TRACHEOSTOMY}

and the number of comorbidities were significant long-term tracheostomy predictors. Further studies to assess outcomes and predictors of tracheostomy subjects are needed.

\section{REFERENCES}

1. Cheung N, Napolitano L. Tracheostomy: epidemiology, indications, timing, technique, and outcomes. Respir Care 2014;59(6):895-915.

2. Rumbak M, Newton M, Truncale T, Schwartz S, Adams J, Hazard P. A prospective, randomized study comparing early percutaneous dilational tracheotomy to prolonged translaryngeal intubation (delayed tracheotomy) in critically ill medical patients. Crit Care Med 2004;32 (8):1689-1694.

3. Bonvento B, Wallace S, Lynch J, Coe B, McGrath B. Role of the multidisciplinary team in the care of the tracheostomy patient. J Multidiscip Healthc 2017;10:391-398.

4. Morris L, Whitmer A, McIntosh E. Tracheostomy care and complications in the intensive care unit. Crit Care Nurse 2013;33(5):18-30.

5. Cox C, Martinu T, Sathy S, Clay A, Chia J, Gray A, et al. Expectations and outcomes of prolonged mechanical ventilation. Crit Care Med 2009;37(11):2888-2894.

6. Engoren M, Arslanian-Engoren C, Fenn-Buderer N. Hospital and long-term outcome after tracheostomy for respiratory failure. Chest 2004;125(1):220-227.

7. Stelfox H, Hess D, Schmidt U. A North American survey of respiratory therapist and physician tracheostomy decannulation practices. Respir Care 2009;54(12):1658-1664.

8. Mitchell R, Hussey H, Setzen G, Jacobs I, Nussenbaum B, Dawson C, et al. Clinical consensus statement: tracheostomy care. Otolaryngol Head Neck Surg 2013;148(1):6-20.

9. Singh R, Saran S, Baronia A. The practice of tracheostomy decannulation-a systematic review. J Intensive Care 2017;5(1).

10. Medeiros G, Sassi F, Lirani-Silva C, Andrade C. Criteria for tracheostomy decannulation: literature review. Codas 2019;31(6):e20180228.

11. Enrichi C, Battel I, Zanetti C, Koch I, Ventura L, Palmer K, et al. Clinical criteria for tracheostomy decannulation in subjects with acquired brain injury. Respir Care 2017;62(10):1255-1263.

12. Choate K, Barbetti J, Currey J. Tracheostomy decannulation failure rate following critical illness: a prospective descriptive study. Aust Crit Care 2009;22(1):8-15.

13. Stelfox H, Crimi C, Berra L, Noto A, Schmidt U, Bigatello L, Hess D. Determinants of tracheostomy decannulation: an international survey. Crit Care 2008;12(1):R26.

14. Peduzzi P, Concato J, Kemper E, Holford T, Feinstein A. A simulation study of the number of events per variable in logistic regression analysis. J Clin Epidemiol 1996;49(12):1373-1379.

15. Choi B, Pak A. Multidisciplinarity, interdisciplinarity, and transdisciplinarity in health research, services, education, and policy: 1. definitions, objectives, and evidence of effectiveness. Clin Invest Med 2006;29(6):351-364.

16. Körner M. Interprofessional teamwork in medical rehabilitation: a comparison of multidisciplinary and interdisciplinary team approach. Clin Rehabil 2010;24(8):745-755.

17. Mah J, Staff I, Fisher S, Butler K. Improving decannulation and swallowing function: a comprehensive, multidisciplinary approach to posttracheostomy care. Respir Care 2017;62(2):137-143.

18. Perin C, Meroni R, Rega V, Braghetto G, Cerri C. Parameters influencing tracheostomy decannulation in patients undergoing rehabilitation af ter severe acquired brain injury (sABI). Int Arch Otorhinolaryngol 2017;21(4):382-389.

19. O'Connor H, Kirby K, Terrin N, Hill N, White A. Decannulation following tracheostomy for prolonged mechanical ventilation. J Intensive Care Med 2009;24(3):187-194.

20. Heidler M, Salzwedel A, Jöbges M, Lück O, Dohle C, Seifert M, et al. Decannulation of tracheotomized patients after long-term mechanical ventilation - results of a prospective multicentric study in German neurological early rehabilitation hospitals. BMC Anesthesiol 2018;18 (1):65.

21. Lima CA, Siqueira TB, Travassos ÉF, Macedo CMG, Bezerra AL, Paiva Júnior MDS, et al. Influence of peripheral muscle strength on the decannulation success rate. Rev Bras Ter Intensiva 2011;23(1):5661.

22. McGrath B, Wallace S. The UK national tracheostomy safety project and the role of speech and language therapists. Curr Opin Otolaryngol Head Neck Surg 2014;22(3):181-187.

23. Schneider H, Hertel F, Kuhn M, Ragaller M, Gottschlich B, Trabitzsch A, et al. Decannulation and functional outcome after tracheostomy in patients with severe stroke (DECAST): a prospective observational study. Neurocrit Care 2017;27(1):26-34.

24. Speed L, Harding K. Tracheostomy teams reduce total tracheostomy time and increase speaking valve use: a systematic review and metaanalysis. J Crit Care 2013;28(2):216.e1-216.e10.

25. Budweiser S, Baur T, Jörres R, Kollert F, Pfeifer M, Heinemann F. Predictors of successful decannulation using a tracheostomy retainer in patients with prolonged weaning and persisting respiratory failure. Respiration 2012;84(6):469-476.

26. Santus P, Gramegna A, Radovanovic D, Raccanelli R, Valenti V, Rabbiosi D, et al. A systematic review on tracheostomy decannulation: a proposal of a quantitative semiquantitative clinical score. BMC Pulm Med 2014;14:201.

27. Alhashemi H. Dysphagia in severe traumatic brain injury. Neurosciences (Riyadh) 2010;15(4):231-236.

28. Caruso P, Albuquerque A, Santana P, Cardenas L, Ferreira J, Prina E, et al. Diagnostic methods to assess inspiratory and expiratory muscle strength. J Bras Pneumol 2015;41(2):110-123.

This article is approved for Continuing Respiratory Care Education credit. For information and to obtain your CRCE

(free to AARC members) visit

www.rcjournal.com

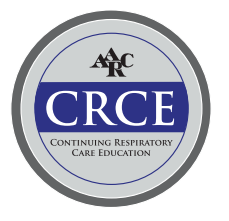

